\title{
Software de diseño de instrucción. Evaluación de su utilidad para diseñar exposiciones educativas en museos
}

\author{
Instructional design software. Evaluation of its usefulness for the \\ design of educational exhibitions in museums
}

RECIBIDO 20/12/2019 ACEPTADO 11/8/2020 PUBLICADO 1/12/2020

\author{
Josep Bosch Bonacasa \\ Universidad de Girona, España
}

u1010545@campus.udg.edu

\section{RESUMEN}

Algunas exposiciones museísticas se diseñan con objetivos educativos. Estas exposiciones, se enmarcan dentro de la educación no formal aunque, si se analizan desde el punto de vista de la instrucción, tienen muchos aspectos en común con las actividades educativas de la educación formal.

Existe software desarrollado para diseñar actividades propias de la educación formal. En cambio, no se ha encontrado software para el diseño de exposiciones educativas en museos.

El objetivo de esta investigación es responder dos preguntas:

1. ¿Puede algún software existente ser utilizado para diseñar exposiciones educativas en museos?

2. ¿Puede algún software existente servir de base para desarrollar un software específico para diseñar exposiciones educativas en museos?

Para responder estas preguntas, en primer lugar, se han seleccionado y definido los conceptos que debería incluir un software para el diseño de exposiciones. Muchos de estos conceptos son compartidos con los utilizados para el diseño de las actividades propias de la educación formal; otros, son específicos y han sido definidos en esta investigación.

En segundo lugar, de entre diecisiete software de diseño de instrucción, se han seleccionado siete que han podido ser instalados y evaluados. A continuación, se ha verificado, uno a uno, si contemplan los conceptos que un software de diseño de exposiciones debería incluir.

Los resultados de la evaluación se presentan en forma de tabla y algunas observaciones. Su análisis ha permitido responder las preguntas formuladas.

De los siete evaluados, uno, Compendium LD, puede ser utilizado para el diseño de exposiciones educativas en museos, aunque con limitaciones. Ninguno de los analizados contempla conceptos importantes como la ubicación de los objetos educativos en el espacio o la descripción de los recorridos del visitante. Compendium LD puede ser, además, un buen punto de partida para desarrollar un software específico.

PALABRAS CLAVE diseño de instrucción, evaluación de software, museos, educación no formal, aprendizaje, instrucción. 


\section{ABSTRACT}

Some museum exhibitions are designed for educational purposes. These exhibitions are part of non-formal education although, if analysed from the point of view of instruction, they have many aspects in common with the educational activities of formal education.

There is software developed to design activities that are proper to formal education. However, no software has been found for the design of educational exhibitions in museums.

The aim of this research is to answer two questions:

1. Can any existing software be used to design educational exhibitions in museums?

2. Can any existing software be used as a basis for developing specific software for designing educational exhibitions in museums?

In order to answer these questions, firstly, the concepts that should be included in software for exhibition design have been selected and defined. Many of these concepts are shared with those used for the design of formal education activities; others are specific and have been defined in this research.

Secondly, from among seventeen instructional design software, seven have been selected that have been able to be installed and evaluated. Next, it has been verified, one by one, if they contemplate the concepts that an exhibition design software should include.

The results of the evaluation are presented in a table and some observations. Their analysis has allowed to answer the questions asked.

Of the seven evaluated, one, Compendium LD, can be used for the design of educational exhibitions in museums, although with limitations. None of those analysed consider important concepts such as the location of educational objects in space or the description of visitor itineraries. Compendium LD can also be a good starting point for developing specific software.

KEYWORDS instructional design, computer software evaluation, museums, non-formal education, learning, instruction.

\section{INTRODUCCIÓN}

\subsection{Objeto de estudio}

Uno de los objetivos del diseño de aprendizaje y del diseño de instrucción es diseñar actividades educativas que faciliten el aprendizaje y la instrucción. Existen matices entre el concepto de diseño de aprendizaje y el de diseño de instrucción (Wasson y Kirschner, 2020), pero en este artículo los consideraremos intercambiables.

El diseño de aprendizaje se interesa por la planificación y el desarrollo de actividades educativas con el objetivo de aumentar el aprendizaje de los estudiantes (Celik y Magoulas, 2016). Intenta definir, también, un marco para describir las actividades de enseñanza y aprendizaje y explorar, como este marco puede ayudar a los instructores a compartir y adoptar buenas prácticas (Dalziel et al., 2016). El diseño de aprendizaje persigue, así, una representación de lo que ocurre en una sesión de aprendizaje cuyo objetivo es alcanzar unos resultados educativos determinados (Laurillard et al., 2018).

Muchos conceptos del diseño de aprendizaje y del diseño de instrucción son aplicables al diseño de exposiciones educativas que se ofrecen en museos.

Los museos tienen potencial para la educación no formal (Mujtaba et al., 2018). Al igual que los materiales de aprendizaje, que están diseñados teniendo presente las necesidades del destinatario, las exposiciones educativas deben diseñarse considerando quien las va a visitar (Hooper-Greenhill, 1999).

Algunas exposiciones comparten muchas características con las actividades de la educación formal: en ambas, por ejemplo, se establecen objetivos educativos y se emplean objetos educativos similares, como 
lecturas, videos o la utilización de computadoras (Bitgood, 2002). Pero las exposiciones educativas en museos tienen unas características diferenciadoras. El visitante, por ejemplo, se desplaza por la exposición utilizando, con frecuencia, todos sus sentidos y su cuerpo. La interacción entre el cuerpo y el entorno es un aspecto importante para el aprendizaje, ya que el cuerpo está en la base del modo en el que experimentamos e interactuamos con el entorno (Koutsabasis y Vosinakis, 2018). Otro ejemplo de característica diferenciadora es que, en una exposición, los objetos educativos se distribuyen en todo el espacio expositivo. En algunos casos, el espacio en el que se desarrolla la actividad educativa incluso puede extenderse más allá de las salas del propio museo (Nisi et al., 2018).

Existe un considerable número de software para auxiliar en el diseño de actividades propias de la educación formal. Este software sería lo que algunos autores, como Sobreira y Tchounikine (2015), llaman learning scenario editor. Sin embargo, no se ha localizado software específicamente desarrollado para auxiliar en el diseño de exposiciones educativas en museos.

Utilizar un software para el diseño de exposiciones permitiría estandarizar su descripción, mejorar el proceso de diseño y también, mediante la evaluación de resultados, identificar patrones de diseño especialmente útiles. Todo ello revertiría en un aumento del aprendizaje por parte de los visitantes.

El objetivo de la investigación es responder dos preguntas:

1. ¿Puede algún software existente ser utilizado para diseñar exposiciones educativas en museos?

2. ¿Puede algún software existente servir de base para desarrollar un software específico para diseñar exposiciones educativas en museos?

\subsection{Fundamentación teórica}

Un conjunto de conceptos y sus relaciones, es un lenguaje. Se han venido desarrollando diferentes representaciones para definir y documentar un diseño de aprendizaje (Celik y Magoulas, 2016).

Educational Modelling Language (EML), que encontramos descrito en Koper y Manderveld (2004), es uno de los lenguajes más conocidos. EML no se centra en ninguna aproximación pedagógica concreta. Integrated Management Systems-Learning Design (IMS-LD), basado en EML, es otro lenguaje muy extendido. IMS-LD, definido por el IMS Global Learning Consortium (2003), tampoco se centra en ninguna aproximación pedagógica concreta. Esta versatilidad permite que estos lenguajes incluyan conceptos que también son adecuados para el diseño de exposiciones museísticas.

Desarrollar un lenguaje común, aplicable a todas las situaciones educativas es, aún, algo que debe ser explorado (Celik y Magoulas, 2016).

Esta investigación contribuye, también, a acercarnos a este lenguaje estándar, lo cual permitiría, además, el análisis de datos, utilizado en educación para analizar aspectos como la retención de los estudiantes, el grado de participación o la interacción (Hernández-Leo et al., 2019).

Se ha venido desarrollando gran cantidad de software para asistir en el diseño de contenidos y también para planificar los objetivos educativos, los métodos de evaluación y otros aspectos del aprendizaje (Prieto et al., 2013). Detrás de cada software de diseño de actividades educativas, subyace una determinada representación, un determinado lenguaje. El exceso de oferta puede dificultar a los investigadores y a los diseñadores localizar el software más adecuado para sus objetivos (Prieto et al., 2013). 


\subsection{Antecedentes}

Los antecedentes a esta investigación los encontramos, sobre todo, en el diseño de aprendizaje en educación formal.

Persico y Pozzi (2015) analizaron el conjunto de investigación reciente en el campo del diseño de aprendizaje, y afirmaron que la mayoría de las investigaciones pueden clasificarse en una de las áreas siguientes: el análisis de las representaciones que pueden ser utilizadas como lenguaje de diseño; las herramientas que pueden ser utilizadas en el proceso de diseño; $y$, finalmente, las aproximaciones metodológicas al diseño de aprendizaje.

Con relación a la representación, al lenguaje, Pozzi et al. (2015) realizaron una revisión sistemática de las representaciones existentes. Figl et al. (2010) analizaron los Visual Instructional Design Languages (VIDL) y las ventajas cognitivas derivadas de la utilización de representaciones graficas de los conceptos. Katagall et al. (2015) trataron los fundamentos teóricos de los mapas conceptuales (un tipo de representación gráfica), destacando su utilidad para describir y comprender problemas complejos, como sería el diseño de instrucción. Chrobak et al (2015) apuntan que los mapas conceptuales pueden desarrollar, además, la creatividad.

Merrill (2001) señaló la importancia de alcanzar un estándar y propuso un vocabulario común que este estándar debería tener.

Hornecker y Buur (2006) analizaron algunas actividades educativas donde el movimiento del individuo y la interactividad tienen un papel importante. Malinverni et al. (2016) investigaron ambientes de aprendizaje en los que existe interacción física del usuario con los objetos educativos.

Merriënboer et al. (2017) destacaron la importancia del espacio físico en el que debe desarrollarse una actividad educativa, aunque no desde el punto de vista de la ubicación de objetos educativos en el espacio, sino más bien en definir unas características funcionales que debería reunir el espacio físico en el que se desarrolla una tarea de este tipo.

Con relación al análisis de herramientas, Dalziel et al. (2016) enumeraron, por orden cronológico de aparición, un gran número de software de diseño de aprendizaje. Pozzi et al. (2020) también enumeraron varias herramientas para el diseño de aprendizaje.

Celik y Magoulas (2016) presentaron una exhaustiva clasificación de software relacionado con el diseño de instrucción. A diferencia de nuestro análisis, solo se analizaron según unas propiedades generales, tales como el lenguaje base que el software utiliza y algunas especificaciones técnicas, sin analizar los conceptos y relaciones que el software contempla.

Britain (2004) analizó una selección de software y planteó la metodología, utilizada en esta investigación, consistente en analizar las entidades, objetos y conceptos desde el punto de vista del usuario, aunque Britain (2004), sobre todo, analizó aspectos técnicos, requerimientos mínimos de los equipos y su funcionalidad principal.

Conscientes del gran número de herramientas disponibles, Integrated Learning Design Environment (ILDE) es una plataforma que integra funciones de colaboración entre diseñadores de contenidos y la posibilidad de representarlos mediante diferentes herramientas de diseño ya existentes (Hernández-Leo et al., 2018).

Con relación a investigaciones relacionadas con las aproximaciones metodológicas, Muñoz-Cristóbal et al. (2018) analizaron el proceso de diseño de contenidos. Lachheb y Boling (2018) preguntando a 100 diseñadores de instrucción, constataron la gran variedad de métodos y herramientas utilizados. 
Finalmente, como antecedentes relacionados con los museos, Gómez-Redondo et al. (2017) describieron un instrumento para el inventario, análisis y posterior evaluación de recursos didácticos de las instituciones museísticas. Fontal et al. (2019) evaluaron el programa educativo de las instituciones museísticas sin centrarse en una actividad educativa concreta. Crack y Cohn (2015) analizaron diferentes métodos de obtención de datos para evaluar el cumplimiento de los objetivos educativos de una exposición, pero no se plantearon estandarizar la descripción de los contenidos como necesidad previa a esta evaluación.

\section{MÉTODO}

Primero se han localizado y descrito los conceptos que un lenguaje para el diseño de exposiciones educativas en museos debería contemplar. Por un lado, se han incorporado los conceptos que permiten definir las actividades propias de la educación formal y que son comunes a una exposición museística; y, por el otro, se han definido los conceptos específicos que debería contemplar un software para el diseño de exposiciones educativas. También se han descrito las relaciones entre estos conceptos. En esta misma sección pueden consultarse la definición de estos conceptos (2.1) y sus relaciones (2.2).

A continuación, se ha buscado un software para el diseño de instrucción. De entre diecisiete software potencialmente útiles, se han seleccionado siete que han sido instalados. Los diecisiete software del conjunto inicial pueden consultarse en la tabla 1 de la sección 2.3.

Posteriormente se ha procedido a evaluar, concepto a concepto, si está contemplado en el software. Finalmente, se han analizado una a una, si las relaciones entre conceptos están contempladas en el software. El resultado, resumido en la tabla 2, puede consultarse en la sección 3.

A partir del resultado de la evaluación se ha procedido a contestar las preguntas definidas en el objetivo de la investigación.

\subsection{Conceptos analizados}

A continuación, se describen los conceptos y sus relaciones. Gran parte de los conceptos aparecen en los diferentes lenguajes ya citados o se encuentran implícitos en el software analizado. Dichos conceptos son definidos adaptándolos a la realidad de una exposición educativa. Por otro lado, cuando el concepto es una aportación de esta investigación a un posible lenguaje para el diseño de exposiciones educativas en museos, se especifica en la descripción del concepto. Estas definiciones se incluyen para saber el concepto o la relación exactamente evaluada. También se incluyen porque son los que deberían incorporarse a un lenguaje estandarizado para el diseño de instrucción.

\subsubsection{Actores}

Actor es cualquier individuo, que, de una u otra forma, interviene en una actividad educativa.

El visitante de una exposición, sea o no estudiante, tiene determinadas características físicas. Estas características permiten la cognición a partir de las que emerge el aprendizaje.

El visitante se desplaza físicamente por el espacio de la exposición y lo hace a lo largo de unos intervalos de tiempo. El visitante, además, ejecuta determinadas acciones, percibe los contenidos de la exposición a través de sus sentidos y los interpreta. 


\subsubsection{Rol, visitante}

El software permite introducir el rol de visitante (asimilable al rol de estudiante).

\subsubsection{Rol, guía}

El software permite introducir, cuando existe, el rol de guía de la exposición (asimilable al rol de profesor).

\subsubsection{Rol, cualquier otro}

Se analiza si el software incorpora la posibilidad de introducir y contemplar otros roles.

\subsubsection{Perfil}

Los visitantes pueden agruparse según determinadas características comunes como la edad, el nivel de estudios o la movilidad. Puede suponerse cierta homogeneidad en el comportamiento de los integrantes de un determinado perfil.

\subsubsection{Grupos}

El software permite la creación de grupos de individuos. Esta opción está especialmente indicada para analizar actividades educativas colaborativas. Los diferentes integrantes del grupo pueden tener diferentes perfiles.

\subsubsection{Objetivos educativos}

Se analiza si el software permite introducir y gestionar los objetivos educativos (goal). Otros nombres tangentes a este concepto y también de amplia difusión, son: objetivo de aprendizaje (learning objective), resultado de aprendizaje (learning outcome), competencias (competencies), objetivos de la instrucción (instruccional objectives) y competencias objetivo (target competencies).

Si consideramos que un individuo es un sistema, el aprendizaje seria la modificación de este sistema mediante las dinámicas propias de la cognición. Por ejemplo, se da la modificación de la memoria declarativa al almacenar hechos o se da la modificación de la memoria procedimental para interiorizar, no necesariamente de manera consciente, secuencias de acciones que el individuo debe ejecutar para, partiendo de un estado inicial, alcanzar un estado deseado. Los objetivos educativos se alcanzan mediante secuencias de acciones.

\subsubsection{Clasificación de los objetivos educativos}

Se analiza si el software permite algún tipo de clasificación de los objetivos educativos, pudiendo ser estos tan variados como recordar un hecho, aprender un procedimiento o adoptar una determinada actitud en relación a una problemática.

\subsubsection{Objetos educativos}

Se analiza si el software permite definir y gestionar objetos educativos (learning objects), es decir, aquello que el visitante puede percibir e interpretar. Un objeto educativo se define mediante el significante, que aporta significado, y por elementos accesorios. 


\subsubsection{Tipo de significante}

Concepto de nueva incorporación. Aquello percibido por nuestro sistema sensorial y que es causante de un significado es un significante. Ejemplos de tipos de significante serían un texto, un olor, una textura o una imagen. Se pueden clasificar los objetos educativos según el tipo de significante.

\subsubsection{Percepción, sentidos}

Concepto de nueva incorporación. Se analiza si el software permite asignar a cada uno de los objetos educativos el sentido que se utiliza para su percepción.

\subsubsection{Conjunto de objetos}

Concepto de nueva incorporación. Un objeto educativo es, con frecuencia, un conjunto de objetos. Un objeto educativo, como un plafón explicativo, está formado, por ejemplo, de imágenes y texto. Podemos definir análisis, como la posibilidad de definir los objetos que componen un objeto; y, síntesis, como la posibilidad, inversa, de asignar un nombre para definir un conjunto de objetos. El software permite definir conjuntos de objetos. Ver también 2.1.9.

\subsubsection{Herramientas}

Aquello que debe proporcionarse a un actor para que pueda ejecutar una acción es una herramienta (tool). El acceso a algunos objetos educativos es mediante la utilización de herramientas. En el caso del museo, una herramienta típica podría ser una audioguía. El contenido de la audioguía es el objeto educativo y la audioguía es la herramienta. Ver también 2.1.9.

\subsubsection{Prerrequisitos}

Los prerrequisitos son aquellos conocimientos que el visitante debe poseer antes de iniciar la actividad. Estos conocimientos previos les permitirán una correcta interpretación de la exposición y también el correcto uso de las herramientas que se utilizan para ejecutar la visita.

\subsubsection{Prerrequisito conocimiento}

Conocimientos generales, declarativos o procedimentales, necesarios para la correcta interpretación de la exposición. Se analiza si estos prerrequisitos están contemplados en el software. Ver también 2.1.7 y 2.1.14.

\subsubsection{Prerrequisito técnico}

Conocimientos o habilidades que los actores deben poseer con relación a las herramientas que deberán utilizar, como por ejemplo, conocer el funcionamiento de una pantalla táctil. Ver también 2.1.13.

\subsubsection{Acciones}

El diseño que consideramos más adecuado para formalizar el contenido de una exposición estaría centrado 
en las acciones ejecutadas por los diferentes roles. Una acción puede definirse como unos atributos en un estado inicial y unos atributos en un estado final.

\subsubsection{Estado inicial}

Se pueden describir los atributos en un estado inicial. Ver también 2.1.17.

\subsubsection{Estado final}

Se pueden describir los atributos en un estado final. Ver también 2.1.17.

\subsubsection{Intervalos de tiempo-duración}

Una acción tiene un inicio en el tiempo y un final. Tiene también una duración. Un vídeo o un audio, presentan unos contenidos de manera secuencial, donde los intervalos de tiempo son conocidos. Otros objetos educativos, como el texto de una cartela, tienen unos intervalos de tiempo menos previsibles. Se analiza si estos intervalos pueden ser definidos. Ver también 2.1.17.

\subsubsection{Secuencia de acciones}

Mediante secuencias de acciones podemos definir, por ejemplo, el recorrido del visitante por la exposición o también la interacción con los elementos de la exposición. Las acciones se ejecutan de forma secuencial. Se analiza si es posible modelar secuencias de acciones. Ver también 2.1.17.

\subsubsection{Bifurcación de acciones}

Es posible modelar una elección entre diferentes acciones. Ver también 2.1.17.

\subsubsection{Unión de acciones}

Es posible modelar que varias acciones son condición para ejecutar otra acción. Ver también 2.1.17.

\subsubsection{Eventos}

Es posible modelar cambios en algún estado de alguna característica en un tiempo determinado.

\subsubsection{Espacio}

Concepto de nueva incorporación. Es posible gestionar una representación del espacio en el que se desarrolla la actividad educativa. Por ejemplo, un mapa de la exposición. El tratamiento del espacio en exposiciones tiene diferencias notables con el tratamiento del espacio en educación formal. De hecho, solo algunos lenguajes hacen referencia al espacio y, cuando lo hacen, son referencias relativas al lugar donde ocurre el aprendizaje, como, por ejemplo, un aula o un laboratorio. Otros autores, utilizan el concepto de ambiente (Koper, 2001). 


\subsubsection{Espacio-recorrido}

Concepto de nueva incorporación. Se analiza si es posible modelar el recorrido del visitante de la exposición. Ver también 2.1.25.

\subsubsection{Espacio, ubicación objetos educativos}

Concepto de nueva incorporación. En exposiciones, los objetos educativos se ubican en el espacio. Es posible representar sobre un plano la ubicación de los objetos educativos. Ver también 2.1.25.

\subsubsection{Métodos de evaluación de resultados}

Se analiza si el software permite una evaluación de resultados, centrando la comparación, mediante algún método de evaluación, entre lo planificado y lo alcanzado.

\subsubsection{Secuencia visita real}

Es posible introducir los datos de visitas reales. Las secuencias de acciones ejecutadas por un visitante.

\subsubsection{Resultados educativos reales}

Con relación a los objetivos educativos, es importante diferenciar entre aquello que eran objetivos y aquello que realmente ha sido alcanzado por el visitante (outcomes). Se analiza si es posible expresar que unos resultados han sido alcanzados o que no lo han sido. Ver también 2.1.7.

\subsubsection{Efectos inesperados}

En algunos casos, pueden aparecer competencias adquiridas pero que no están directamente relacionadas con los objetivos del diseño (side-effects). Ver también 2.1.7.

\subsubsection{Producciones del visitante}

Un resultado tangible (learning output) producido por el visitante, como por ejemplo un objeto, texto o un dibujo.

\subsection{Relaciones entre conceptos}

A continuación, se definen las relaciones entre los conceptos introducidos en 2.1. Consideramos que un software para el diseño de exposiciones educativas en museos debería tenerlas implementadas. Se trata de información que debería quedar almacenada en el software de manera explícita.

\subsubsection{Acción-objetivos educativos}

Se analiza si es posible identificar las acciones que permiten alcanzar cada uno de los objetivos educativos. Una misma acción puede ser ejecutada para alcanzar varios objetivos educativos. 


\subsubsection{Acción-rol actor}

Se pueden identificar las acciones que realiza cada uno de los actores según su rol. Cada acción tiene asociado el rol que la ejecuta.

\subsubsection{Acción-objeto educativo}

El software permite expresar los objetos educativos utilizados en cada una de las acciones.

\subsubsection{Acción-herramientas}

El software contempla, de forma explícita, las herramientas utilizadas para ejecutar cada acción.

\subsubsection{Acción-prerrequisito}

El software permite expresar la relación entre cada una de las acciones y cada uno de los prerrequisitos necesarios para ejecutarlas. Una misma acción puede tener varios prerrequisitos. Un mismo prerrequisito puede ser necesario para ejecutar varias acciones.

\subsubsection{Acciones-producciones}

El software permite expresar las producciones que resultan de una acción.

\subsubsection{Acciones-resultados reales}

Se pueden localizar las acciones que contribuyen a un resultado educativo real.

\subsubsection{Acciones-resultado educativo inesperado}

Es posible identificar las acciones que contribuyen a un resultado educativo inesperado.

\subsubsection{Prerrequisito conocimiento-objetivo educativo}

Es posible expresar que prerrequisitos son condición para alcanzar cada uno de los objetivos educativos. Un objetivo educativo puede tener varios prerrequisitos.

\subsubsection{Prerrequisito técnico-herramienta}

Prerrequisito de conocimiento procedimental. Es posible expresar, para cada una de las herramientas que deben utilizarse en la visita, los prerrequisitos técnicos necesarios para utilizarlas.

\subsubsection{Prerrequisito conocimiento-perfil}

Se pueden definir cada uno de los prerrequisitos de conocimiento que deben tener los integrantes de un perfil. 


\subsubsection{Prerrequisito técnico-perfil}

Se pueden definir cada uno de los prerrequisitos técnicos que deben tener los integrantes de un perfil.

\subsubsection{Perfil-objetivo educativo}

Es posible especificar los objetivos educativos para cada uno de los perfiles.

\subsubsection{Perfil-recorrido teórico por el espacio}

Es posible describir el recorrido teórico, o más probable, de un perfil de visitante a través del espacio de la exposición.

\subsubsection{Perfil-secuencia teórica de acciones}

Es posible representar la secuencia de acciones teórica que los visitantes pertenecientes a un perfil llevarán a cabo durante la ejecución de la visita a la exposición.

\subsubsection{Individuo-recorrido real en el espacio}

Es posible definir el recorrido real de uno o varios visitantes concretos. Es posible comparar el recorrido teórico del perfil con el recorrido real del visitante.

\subsubsection{Individuo-secuencia real de acciones}

Es posible introducir la secuencia de acciones reales ejecutadas por el visitante. Es posible comparar la secuencia teórica del diseño con la secuencia real del visitante.

\subsubsection{Individuo-objetivo educativo}

Existe un método de evaluación. Es posible evaluar el número de objetivos alcanzados por un individuo.

\subsubsection{Individuo-resultados inesperados}

Es posible introducir los resultados inesperados de un individuo.

\subsubsection{Individuo-producciones}

Es posible introducir las producciones de un individuo determinado. Ver también 2.1.32.

\subsubsection{Objetivo educativo-objeto educativo}

Es posible identificar los objetos educativos que contribuyen a alcanzar cada uno de los objetivos educativos. 


\subsubsection{Objetivo educativo-herramienta}

Es posible identificar las herramientas necesarias para alcanzar cada uno de los objetivos educativos.

\subsubsection{Objeto educativo-espacio}

Es posible identificar la ubicación de los objetos educativos en el espacio de la exposición.

\subsubsection{Representación gráfica}

El software incorpora algún tipo de representación gráfica de conceptos y sus relaciones.

\subsection{Selección de software a evaluar}

Una búsqueda preliminar permitió localizar diecisiete candidatos a ser incluidos en la evaluación. En la tabla 1 pueden observarse los candidatos iniciales y si se incorporaron al análisis. Los software candidatos fueron: Cadmos (Katsamani y Retalis, 2013) que ya no recibe soporte en la actualidad y esto imposibilitó su instalación; Collage, Reload y WebCollage (Hernández-Leo et al., 2010), instalable, son tres proyectos muy interrelacionados; Compendium LD (Brasher et al., 2008), instalable; CPM-TOOL (Laforcade, 2005), se trataba de un prototipo; Edit (Bafail et al., 2017), centrado en medir la calidad de un diseño; Euterpe (Welie et al., 1998), instalable; GLUE!-PS (Prieto et al., 2011), centrado en traducir diseños de otras herramientas a lenguaje interpretable por un virtual learning environment (VLE); Open Graphical Learning Modeller (OGML),

TABLA 1. Software seleccionado para su evaluación

\begin{tabular}{cc}
\hline SOFTWARE & SELECCIONADO \\
\hline Cadmos & $\checkmark$ \\
\hline Collage-Reload-WebCollage & $\checkmark$ \\
\hline Compendium LD & \\
\hline CPM-TOOL & $\checkmark$ \\
\hline Edit & \\
\hline Euterpe & $\checkmark$ \\
\hline GLUE!-PS & $\checkmark$ \\
\hline LAMS & \\
\hline LdShake & $\checkmark$ \\
\hline LDTool & \\
\hline Learning Design Studio & $\checkmark$ \\
\hline Learning Designer (LDSE) & \\
\hline LPCEL & \\
\hline Open Graphical Learning \\
Modeller
\end{tabular}
localizado en Derntl (2015), instalable; LAMS (Dalziel, 2006), un Learning Management System (LMS); LdShake (Hernández-Leo et al., 2011), plataforma de intercambio de diseños integrado en Collage; LDTool (Agostinho, 2011), funciona online; Learning Design Studio (Mor y Mogilevsky, 2013), centrado en educación formal; Learning Designer (LDSE) de Laurillard et al. (2013), no ha habido continuidad en su desarrollo pero aún es posible localizar el software; LPCEL (Torres et al., 2014), era versión web que no recibe soporte en la actualidad; ReCourse (Griffiths et al., 2009), software ya no disponible que, posiblemente, ya no recibe soporte en la actualidad; ScenEdit (Emin et al., 2010), software ya no disponible, posiblemente, sin continuidad en su desarrollo; Telos MOT+LD (Paquette y Léonard, 2008), software no disponible que, posiblemente, tampoco recibe soporte en la actualidad.

Los no seleccionados son proyectos que no reciben ningún soporte en la actualidad, proyectos que no se desarrollaron completamente o que no con- 
templan conceptos considerados clave. Los seleccionados son aquellos que reciben algún tipo de soporte o que aún es posible localizar e instalar para analizarlos en profundidad.

\section{RESULTADOS}

En la tabla 2 se presentan los resultados de la evaluación.

TABLA 2. Conceptos y relaciones contemplados por el software analizado

\begin{tabular}{|c|c|c|c|c|c|c|c|}
\hline CONCEPTOS Y RELACIONES & $\begin{array}{l}\text { 亗 } \\
\text { ப் }\end{array}$ & 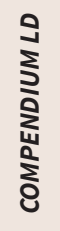 & $\begin{array}{l}\text { U. } \\
\text { 岕 } \\
\text { S. }\end{array}$ & $\begin{array}{l}\text { 임 } \\
\text { 잉 }\end{array}$ & $\sum_{0}$ & $\sum_{\Sigma}^{n}$ & 岃 \\
\hline Actores & $\checkmark$ & $\checkmark$ & $\checkmark$ & $\checkmark$ & $\checkmark$ & $\checkmark$ & $\checkmark$ \\
\hline Rol, visitante (estudiante) & $\checkmark$ & $\checkmark$ & $\checkmark$ & $\checkmark$ & $\checkmark$ & $\checkmark$ & $\checkmark$ \\
\hline Rol, guía (profesor) & $\checkmark$ & $\checkmark$ & $\checkmark$ & $\checkmark$ & $\checkmark$ & $\checkmark$ & $\checkmark$ \\
\hline Rol, cualquier otro rol & & $\checkmark$ & $\checkmark$ & & $\checkmark$ & & \\
\hline Perfil & & $\checkmark$ & $\checkmark$ & & $\checkmark$ & $\checkmark$ & \\
\hline Grupos & & $\checkmark$ & $\checkmark$ & & & $\checkmark$ & \\
\hline Objetivos educativos & $\checkmark$ & $\checkmark$ & & $\checkmark$ & $\checkmark$ & $\checkmark$ & $\checkmark$ \\
\hline Clasificación de objetivos educativos & $\checkmark$ & & & & & & $\checkmark$ \\
\hline Objetos educativos & $\checkmark$ & $\checkmark$ & $\checkmark$ & $\checkmark$ & $\checkmark$ & $\checkmark$ & $\checkmark$ \\
\hline \multicolumn{8}{|l|}{ Tipo de significante } \\
\hline \multicolumn{8}{|l|}{ Percepción, sentidos } \\
\hline Conjunto de objetos & $\checkmark$ & $\checkmark$ & $\checkmark$ & & $\checkmark$ & $\checkmark$ & $\checkmark$ \\
\hline Herramientas & $\checkmark$ & $\checkmark$ & & & $\checkmark$ & $\checkmark$ & \\
\hline Prerrequisitos & $\checkmark$ & $\checkmark$ & & & $\checkmark$ & $\checkmark$ & \\
\hline Prerrequisito conocimiento & $\checkmark$ & $\checkmark$ & & & $\checkmark$ & $\checkmark$ & \\
\hline Prerrequisito técnico & $\checkmark$ & $\checkmark$ & & & $\checkmark$ & $\checkmark$ & \\
\hline Acciones & $\checkmark$ & $\checkmark$ & $\checkmark$ & $\checkmark$ & $\checkmark$ & $\checkmark$ & \\
\hline Estado inicial & & & $\checkmark$ & & & & \\
\hline Estado final & & & $\checkmark$ & & & & \\
\hline Intervalos de tiempo, duración & $\checkmark$ & & $\checkmark$ & & $\checkmark$ & $\checkmark$ & $\checkmark$ \\
\hline Secuencia de acciones & $\checkmark$ & $\checkmark$ & $\checkmark$ & & $\checkmark$ & $\checkmark$ & \\
\hline Bifurcación de acciones & & $\checkmark$ & $\checkmark$ & & $\checkmark$ & $\checkmark$ & \\
\hline Unión de acciones & & $\checkmark$ & $\checkmark$ & & $\checkmark$ & $\checkmark$ & \\
\hline Eventos & & & $\checkmark$ & & & & \\
\hline Espacio & & & & & & & \\
\hline
\end{tabular}


Innoeduca. International Journal of Technology and Educational Innovation

Bosch Bonacasa, J.

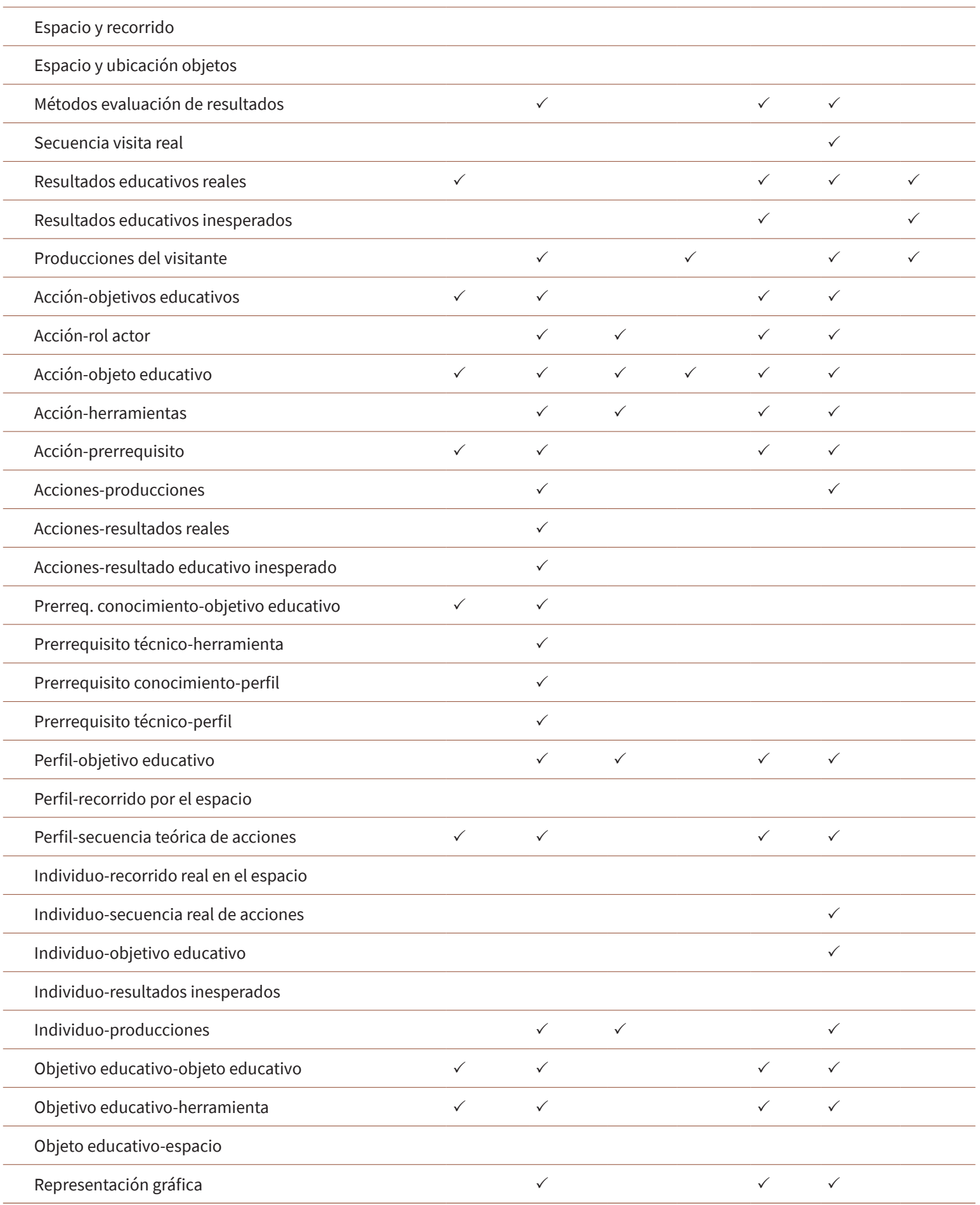

Un $\checkmark$ en la tabla indica que el software contempla el concepto o la relación entre conceptos tal como se ha definido en la sección 2.

\subsection{Observaciones}

Compendium $L D$ es un software que utiliza representación gráfica; permite la creación de nuevos tipos de conceptos en forma de nodos y nombrar las relaciones entre estos, especificando su significado conceptual. 
Su versatilidad lo convierte en el software que más conceptos contempla. Compendiun está publicado bajo licencia Lesser General Public License (LGPL).

$L D$ tool, integrada en la plataforma ILDE, puede utilizarse online.

Collage es el resultado de la integración de diversos programas.

Euterpe es un gestor de tareas no específicamente desarrollado para el diseño de instrucción. Es un software que permite analizar y describir ambientes en los que existe cooperación entre individuos.

Open Graphical Learning Modeller (OGLM) está muy centrado en educación formal. Este hecho dificulta su posible aplicación para el diseño de contenidos educativos para exposiciones. En este artículo se considera OGLM como representativo de todo el software desarrollado con lenguaje IMS-LD. OGLM permite desarrollar contenidos exportables a otros Learning Management System (LMS), como Moodle.

LDSE se centra en la clasificación de los objetivos educativos.

LAMS es también un LMS. Por lo tanto, se centra en la gestión de actividades propias de la educación formal.

\section{DISCUSIÓN}

Los softwares analizados, basados en algunos de los lenguajes más extendidos, no incorporan los mismos conceptos y relaciones. Del análisis de resultados se constata, igual que hizo Anido-Rifón et al. (2014) al referirse a los lenguajes, la coexistencia, aún, de diferentes intentos de alcanzar un estándar llevados a cabo por diferentes grupos de investigadores. Podemos afirmar, así, que sigue viva la necesidad, apuntada por Mor y Craft (2012) y también por Dalziel et al. (2016), de desarrollar un lenguaje común.

A partir del análisis de los resultados también se puede afirmar, al igual que Britain (2004), que, aunque IMS-LD, lenguaje utilizado por OGLM, puede considerarse un buen marco para el diseño de aprendizaje, representa solo uno de los posibles enfoques y hay muchos otros que también pueden utilizarse. De hecho, el software más adaptable y que contempla más conceptos y relaciones, Compendium LD, no está basado directamente en el lenguaje IMS-LD.

Se observa también, igual que en Mor y Craft (2012), que para el diseño de contenidos educativos ninguna herramienta contempla todos los requerimientos en todas las situaciones educativas. Los resultados confirman también, en la línea de lo apuntado por Prieto et al. (2013), que existe una gran dificultad en modelizar el aprendizaje y la instrucción mediante software.

Se observa también, coincidiendo con Anido-Rifón et al. (2014), que las herramientas y sus lenguajes subyacentes podrían ser suficientes para modelar la educación en algunos contextos pero no serían suficientemente expresivas para modelar actividades educativas en otros contextos. El diseño de exposiciones museísticas sería uno de estos contextos en los que el software analizado no es completamente adecuado.

\section{CONCLUSIONES}

La investigación permite responder las preguntas formuladas. En relación a la primera, ningún software analizado se adapta completamente al diseño de exposiciones educativas: ninguno contempla todos los conceptos y relaciones definidos en esta investigación; ninguno permite, por ejemplo, definir la distribución de los objetos educativos en el espacio expositivo o los recorridos de los visitantes a través de la exposición. La explicación es que el desarrollo de la mayoría de los lenguajes y softwares para el diseño de actividades 
educativas, han sido desarrollados pensando en la educación formal, más aún, en el diseño de contenidos compatibles con algún Learning Management System (LMS).

Compendium $L D$, debido a su versatilidad, que permite la creación de nuevos tipos de nodos y nuevas relaciones entre nodos y su representación gráfica, es el software que mejor se adapta a las necesidades del diseño de exposiciones educativas. Podemos concluir igual que Figl et al. (2010), que utilizar una representación gráfica es determinante. Gray y Boling (2015) también destacan la importancia de utilizar una correcta representación gráfica y esquemática, ya que alcanzar un estándar que incluya una correcta representación gráfica, sería muy beneficioso para el diseño de actividades educativas formales e imprescindible para el diseño de exposiciones educativas en museos.

En relación con la segunda pregunta, Compendium $L D$, un software con licencia $L G P L$, podría servir de base para desarrollar un software específico para el diseño de exposiciones educativas en museos. Sería necesario incorporar todos los conceptos nuevos y relaciones definidas en esta investigación. Desarrollar una nueva aplicación a partir de Compendium LD es una opción que no debe descartarse.

No existe, aún, un lenguaje estandarizado que permita modelar todas las situaciones de aprendizaje e instrucción. Se puede concluir, también, al igual que Dalziel et al. (2016) que el solo hecho de intentar desarrollar un sistema con una notación general y los conceptos bien definidos, tal como hemos hecho en esta investigación, revierte en una mejora en la comprensión de los procesos de enseñanza y aprendizaje. Así, esta investigación cumple los objetivos y contribuye, también, a acercarnos a un lenguaje estandarizado.

\section{REFERENCIAS}

Agostinho, S. (2011). The use of a visual learning design representation to support the design process of teaching in higher education. Australasian Journal of Educational Technology, 27(6), 961-978.

Anido-Rifón, L. E., Fernández-Iglesias, J. M., Caeiro-Rodríguez, M. Santos-Gago, J. M., Llamas-Nistal, M., Álvarez, L., y Míguez, R. (2014). Standardization in computer-based education. Computer Standards \& Interfaces, 36(3), 604-625.

Bafail, A., Tepper, J., Liggett, A., y Banakhr, F. (2017). EDIT: An educational design intelligence tool for supporting design decisions. International Journal for Infonomics, 10(2), 13071315. https://doi.org/10.20533/iji.1742.4712.2017.0160

Bitgood, S. (2002). Environmental psychology in museums, zoos, and other exhibition centers. En R. Bechtel y A. Churchman (Eds.), Handbook of Environmental Psychology (pp. 461-480). John Wiley \& Sons.

Brasher, A., Conole, G., Cross, S., Weller, M., Clark, P., y White, J. (2008). CompendiumLD - a tool for effective, efficient and creative learning design. En J. Lams (Ed.), Proceedings of the 2008 European LAMS Conference (pp. 78-87). LAMS.
Britain, S. (2004). A Review of learning design: Concept, specifications and tools. Bolton Institute of Higher Education.

Celik, D., y Magoulas, G.D. (2016). A review, timeline, and categorization of learning design tools. En D. Chiu, I. Marenzi, U. Nanni, M. Spaniol y M.Temperini (Eds.), Lecture Notes in Computer Science: Vol. 10013. Advances in Web-Based Learning -ICWL 2016 (pp. 3-13). Springer. https://doi. org/10.1007/978-3-319-47440-3_1

Chrobak, R., García, P., y Prieto, A.B. (2015). Creatividad, mapas conceptuales y TIC en educación. Edmetic, Revista de Educación Mediática y TIC. 4(1), 78-94. https://doi.org/10.21071/ edmetic.v4i1.2900

Crack, A., y Cohn, S. (2015). Data collection methods for evaluating museum programs and exhibitions. Journal of Museum Education, 40(1), 27-36.

Dalziel, J. (2006). Lessons from LAMS for IMS learning design. En R. Kinshuk, P. Koper, P. Kommers, Kirschner, D. Sampson y W. Didderen (Eds.), ICALT 2006. Proceedings of the 6th IEEE International Conference on Advanced Learning Technologies (pp. 
1101-1102). IEEE Computer Society. https://doi.org/10.1109/ ICALT.2006.220

Dalziel, J., Conole, G., Wills, S., Walker, S., Bennett, S., Dobozy, E., y Bower, M. (2016). The Larnaca declaration on learning design. Journal of Interactive Media in Education, 1(7), 1-24. https://doi.org/10.5334/jime.407

Derntl, M. (2015). OpenGLM: integrating open educational resources in IMS learning design authoring. En N. Richard y M. Sharples (Eds.), Technology enhanced learning: Vol. 7. The art \& science of learning design (pp. 157-168). Sense Publishers. https://doi.org/10.1007/978-94-6300-103-8_4

Emin, V., Pernin, J.-P., y Aguirre, J.L. (2010). ScenEdit: an intention-oriented authoring environament to design learning scenarios. En M. Wolpers, P.A. Kirschner, M. Scheffel, S. Lindstaedt y V. Dimitrova (Eds.), Lecture Notes in Computer Science: Vol. 6383. Sustaining TEL: From Innovation to Learning and Practice. EC-TEL 2010 (pp. 626-631). Springer. https://doi. org/10.1007/978-3-642-16020-2_65

Figl, K., Derntl, M., Caeiro, M., y Botturi, L. (2010). Cognitive effectiveness of visual instructional design languages. Journal of Visual Languages and Computing, 21(6), 359-373. https://doi. $\operatorname{org} / 10.1016 /$ j.jvlc.2010.08.009

Fontal, O., García, S., Arias, B., y Arias, V. (2019). Evaluación de la calidad de programas de educación patrimonial: construcción y calibración de la escala Q-Edutage. Revista de Psicodidactica 24(1), 31-38. https://doi.org/10.1016/j.psicod.2018.07.003

Gómez-Redondo, C., Calaf, R., y Merillas, O. (2017). Diseño de un instrumento de análisis para recursos didácticos patrimoniales. Cadmo 25(1), 63-80. https://doi.org/10.3280/CAD2017001008

Gray, C., y Boling, E. (2015). Designerly Tools, Sketching, and Instructional Designers and the Guarantors of Design. En B. Hokanson, G. Clinton y M. Tracey (Eds.), The Design of Learning Experience. Educational Communications and Technology: Issues and Innovations (pp. 109-126). Springer. https://doi. org/10.1007/978-3-319-16504-2_8

Griffiths, D., Beauvoir, P., Liber, O., y Barrett-Baxendale, M. (2009) From reload to recourse: learning from IMS learning design implementations. Distance Education, 30(2), 201-222. https:// doi.org/10.1080/01587910903023199
Hernández-Leo, D., Asensio-Pérez, J. I., Derntl, M., Pozzi, F., Chacón, J., Prieto, L. P., y Persico, D. (2018). An integrated environment for learning design. Frontiers in ICT, 5(9),1-19. https://doi.org/10.3389/fict.2018.00009

Hernández-Leo, D., Jorrín-Abellán, I.M., Villasclaras-Fernández, E.D., Asensio-Pérez, J.I., y Dimitriadis, Y. (2010). A multicase study for the evaluation of a pattern-based visual design process for collaborative learning. Journal of Visual Languages and Computing, 21(6), 313-331. https://doi.org/10.1016/j. jvlc.2010.08.006

Hernández-Leo, D., Martinez-Maldonado, R., Pardo, A., MuñozCristóbal, J. A., y Rodríguez-Triana, M. J. (2019). Analytics for learning design: A layered framework and tools. British Journal of Educational Technology, 50(1), 139-152. https://doi. org/10.1111/bjet.12645

Hernández-Leo, D., Romeo, L., Carralero, M. A., Chacón, J., Carrió, M., Moreno, P., y Blat, J. (2011). LdShake: learning design solutions sharing and co-edition. Computers and education, 57(4), 2249-2260. https://doi.org/10.1016/j.compedu.2011.06.016 Hooper-Greenhill, E. (1999). Education, communication and interpretation: towards a critical pedagogy in museums. En E. Hooper-Greenhill (Ed.), The educational role of the Museum (pp. 3-27). Routledge.

Hornecker, E., y Buur, J. (2006). Getting a grip on tangible interaction: A framework on physical space and social interaction. En R. Grinter, T. Rodden, P. Aoki, E. Cutrell, R. Jeffries y G. Olson (Eds.), CHI 2006 Conference on Human Factors in Computing Systems (pp. 437-446). ACM. https://doi. $\operatorname{org} / 10.1145 / 1124772.1124838$

IMS Global Learning Consortium, Inc. (2003). Learning Design Best Practice and Implementation Guide. https://www.imsglobal. org/learningdesign/index.html

Katagall, R., Dadde, R., Goudar, R. H., y Rao, S. (2015). Concept mapping in education and semantic knowledge representation: An Illustrative Survey. Procedia Computer Science 48, (International Conference on Intelligent Computing, Communication \& Convergence, ICCC-2014), 638-643. https://doi. org/10.1016/j.procs.2015.04.146

Katsamani, M., y Retalis, S. (2013). Orchestrating learning activities using the CADMOS learning design tool. Research in Learning Technology, 21. https://doi.org/10.3402/rlt.v21i0.18051 
Koper, R. (2001). Modelling units of study from a pedagogical perspective: the pedagogical metamodel behind EML. http://eml. ou.nl/introduction/docs/ped-metamodel.pdf

Koper, R., y Manderveld, J. (2004). Educational modelling language: modelling reusable,interoperable, rich and personalised units of learning. British Journal of Educational Technology 35(5), 537-551. https://doi.org/10.1111/j.0007$1013.2004 .00412 . x$

Koutsabasis, P., y Vosinakis, S. (2018). Kinesthetic interactions in museums: conveying cultural heritage by making use of ancient tools and (re-) constructing artworks. Virtual Reality, 22, 103-118. https://doi.org/10.1007/s10055-017-0325-0

Lachheb, A., y Boling, E. (2018). Design tools in practice: instructional designers report which tools they use and why. Journal of Computing in Higher Education 30(1), 34-54. https://doi. org/10.1007/s12528-017-9165-x

Laforcade, P. (2005). Towards a UML-based Educational Modeling Language. En P. Goodyear, D.G. Sampson, D. Jin-Tan Yang, T. Okamoto, R. Hartley y N.-S.Chen

(Eds.), Proceedings - 5th IEEE International Conference on Advanced Learning Technologies, ICALT 2005 (pp. 855-859). IEEE. https://doi.org/10.1109/ICALT.2005.288

Laurillard, D., Charlton, P., Craft, B., Dimakopoulos, D., Ljubojevic, D., Magoulas, G., y Whittlestone, K. (2013). A constructionist learning environment for teachers to model learning designs. Journal of Computer Assisted Learning, 29, 15-30. https://doi. $\operatorname{org} / 10.1111 / j .1365-2729.2011 .00458 . x$

Laurillard, D., Kennedy, E., Charlton, P., Wild, J., y Dimakopoulos, D. (2018). Using technology to develop teachers as designers of TEL: Evaluating the learning designer. British Journal of Educational Technology, 49(6), 1044-1058. https://doi. org/10.1111/bjet.12697

Malinverni, L., Schaper, M.-M., y Pares, N. (2016). An evaluationdriven design approach to develop learning environments based on full-body interaction. Educational Technology Research and Development, 64, 1337-1360. https://doi. org/10.1007/s11423-016-9468-z

Merriënboer, J.J.G., McKenney, S., Cullinan, D., y Heuer, J. (2017). Aligning pedagogy with physical learning spaces. European Journal of Education 52(3), 253-267. https://doi.org/10.1111/ ejed.12225
Merrill, M.D. (2001). Components of instruction toward a theoretical tool for instructional design. Instructional Science, 29(4-5), 291-310. https://doi.org/10.1023/A:1011943808888

Mor, Y., y Craft, B. (2012). Learning design: reflections upon the current landscape. Research in Learning Technology, 20. https:// doi.org/10.3402/rlt.v20i0.19196

Mor, Y., y Mogilevsky, O. (2013). Learning Design Studio: Educational practice as design inquiry of learning. En D. Hernández-Leo, T. Ley, R. Klamma y A. Harrer (Eds.), Lecture Notes in Computer Science: Vol. 8095. Scaling up Learning for Sustained Impact. Proceedings of the ECTEL 2013 (pp. 233-245). Springer. https://doi.org/10.1007/978-3-642-40814-4_19

Mujtaba, T., Lawrence, M., Oliver, M., y Reiss, M. J. (2018). Learning and engagement through natural history museus. Studies in Science Education, 54(1), 41-67. https://doi.org/10.1080/0305 7267.2018 .1442820

Muñoz-Cristóbal, J.A., Hernández-Leo, D., Carvalho, L., MartinezMaldonado, R., Thompson, K., Wardak, D., y Goodyear, P. (2018). 4FAD: A framework for mapping the evolution of artefacts in the learning design process. Australasian Journal of Educational Technology, 34(2), 16-34. https://doi. org/10.14742/ajet.3706

Nisi, V., Dionisio, M., Barretoa, M., y Nunesa, N. (2018). A mixed reality neighborhood tour: Understanding visitor experience and perceptions. Entertainment Computing, 27, 89-100. https://doi.org/10.1016/j.entcom.2018.04.002

Paquette, G., y Léonard, M. (2008). A visual ontology-driven LD editor and player: Application to the planet game case study. Journal of Interactive Media in Education, 2, art.23. https:// doi.org/10.5334/2008-25

Persico, D., y Pozzi, F. (2015). Informing learning design with learning analytics to improve teacher inquiry. British Journal of Educational Technology, 46(2), 230-248. https://doi. org/10.1111/bjet.12207

Pozzi, F., Persico, D., y Earp, J. (2015). A multi-dimensional space for learning design representations and tools. En N. Richard y M. Sharples (Eds.), Technology enhanced learning: Vol. 7. The art \& science of learning design (pp. 49-62). Sense Publishers. https://doi.org/10.1007/978-94-6300-103-8_4

Pozzi, F., Asensio-Perez, J.I., Ceregini, A., Dagnino, F.M., Dimitriadis, Y, y Earp, J. (2020). Supporting and representing Learning 
Design with digital tools: in between guidance and flexibility. Technology, Pedagogy and Education, 29(1), 109-128. https:// doi.org/10.1080/1475939X.2020.1714708

Prieto, L.P., Asensio-Pérez, J.I., Dimitriadis, Y., Gómez-Sánchez, E., y Muñoz-Cristóbal, J.A. (2011). GLUE!-PS: A multi-language architecture and data model to deploy TEL designs to multiple learning environments. En C.D. Kloos, D. Gillet, R.M. Crespo-García, F. Wild y M. Wolpers (Eds.), Lecture Notes in Computer Science: vol. 6964.Towards Ubiquitous Learning. EC-TEL 2011 (pp. 285-298). Springer. https://doi.org/10.1007/978-3642-23985-4_23

Prieto, L., Dimitriadis, Y., Craft, B., Derntl, M., Émin, V., Katsamani, M., y Villasclaras, E. (2013). Learning design Rashomon II: exploring one lesson through multiple tools. Research in Learning Technology, 21. https://doi.org/10.3402/rlt.v21i0.20057

Sobreira, P., y Tchounikine, P. (2015). Table-based representations can be used to offer easy-to-use, flexible, and adaptable learning scenario editors. Computers \& Education, 80, 15-27. https://doi.org/10.1016/j.compedu.2014.08.002

Torres, J., Resendiz, J., Aedo, I., y Dodero, J.M. (2014). A modeldriven development approach for learning design using the LPCEL Editor. Journal of King Saud University - Computer and Information Sciences, 26(1), 17-27. https://doi.org/10.1016/j. jksuci.2013.10.004

Wasson, B., y Kirschner, P.A. (2020). Learning Design: European Approaches. Association for Educational Comunications and Technology. Springer. https://doi.org/10.1007/s11528-02000498-0

Welie, M., Veer, G., y Eliëns, A. (1998). Euterpe -Tools support for analyzing cooperative environments. En T.R.G. Green, L. Bannon, C.P. Warren y J. Buckkleys (Eds.), Ninth European Conference on Cognitive Ergonomics, (pp. 25-30). INRIA. 